\title{
Avaliação química de macronutrientes e minerais de dietas enterais artesanais utilizadas em terapia nutricional domiciliar no sistema único de saúde
}

\section{Chemical evaluation of macronutrients and minerals from handmade enteral diets used in homecare nutritional therapy in the brazilian national health system}

\author{
Ann Kristine Jansen ${ }^{1}$ \\ Simone de Vasconcelos Generoso \\ Lígia Amanda Ventura de Oliveira Miranda² \\ Eduarda Guimarães Guedes ${ }^{2}$ \\ Gilberto Simeone Henriques' \\ ' Departamento de Nutrição, Escola de \\ Enfermagem. Universidade Federal de Minas \\ Gerais. Belo Horizonte, MG, Brasil. \\ ${ }^{2}$ Departamento de Nutrição, Curso de Nutrição. \\ Universidade Federal de Minas Gerais. Belo \\ Horizonte, MG, Brasil. \\ Apoio financeiro: Fundação de Amparo à \\ Pesquisa de Minas Gerais (FAPEMIG) \\ Edital 07/2012, Processo No. CDS-APQ-02570-12 \\ Ministério da Saúde/Organização Pan-Americana \\ da Saúde, Carta Acordo BR/LOA/1300093 \\ Correspondência / Correspondence \\ Ann Kristine Jansen \\ UFMG - Escola de Enfermagem \\ Av. Professor Alfredo Balena, 190, sala 314, \\ Santa Efigênia \\ 30130-100, Belo Horizonte, MG, Brasil \\ E-mail: akj@enf.ufmg.br
}

\section{Resumo}

Objetivo: este estudo objetivou avaliar quimicamente a composição de macronutrientes e minerais de dietas enterais artesanais prescritas para terapia nutricional domiciliar. Métodos: Foram coletadas fichas técnicas de dietas enterais artesanais padrão e padrão sem lactose, prescritas na alta hospitalar por hospitais públicos e pelo Núcleo de Apoio à Saúde da Família. Após preparo das formulações e avaliação de viscosidade, estabilidade, odor, cor e custo, foram selecionadas dietas de cinco locais, sendo agrupadas de acordo com as semelhanças na composição em Grupo Ae Grupo B, e encaminhadas para análise química de macronutrientes e minerais. Os resultados foram comparados com as necessidades nutricionais propostas pelas Recomendações Dietéticas de Referência para homens de 51-70 anos. Resultados e Discussão: As dietas analisadas apresentaram distribuição normoproteica, normolipídica e normoglicídica. A quantidade total de fibra alimentar nas dietas do Grupo A ficou entre $12 \mathrm{~g}$ e $15 \mathrm{~g}$, com $48 \%$ de fibra solúvel. No Grupo B, a fibra alimentar foi de 3,40 $\mathrm{g}$, e na dieta padrão sem lactose, entre 6 e $8 \mathrm{~g}$, apresentando $65 \%$ de fibra solúvel. Quanto aos minerais, todas as formulações estavam adequadas em ferro e a maioria, em cálcio, zinco, fósforo, cobre e sódio. Nenhuma formulação avaliada encontrou valores adequados de potássio e magnésio. Observou-se ainda baixa adequação de muitos minerais nas formulações de $1.200 \mathrm{Kcal}$. Conclusão: As formulações analisadas têm composição de macronutrientes adequada, necessitando de ajustes em fibras. Quanto aos minerais, aqueles deficientes precisam ser suplementados enquanto as adequações dietéticas não forem realizadas.

Palavras-chave: Segurança Alimentar e Nutricional. Assistência Domiciliar. Nutrição Enteral. Macronutrientes. Composição Química. Sistema Único de Saúde. Adultos. 


\section{Abstract}

Objective: This study aimed to evaluate the chemical composition of nutrients and minerals from handmade enteral diets prescribed in enteral homecare. Methods: Datasheets from standard diets and lactose-free diets prescribed at discharge from public hospitals and by the Support Center for Family Health were collected. After the preparation of diets and assessment of viscosity, stability, odor, color and cost, five diets were selected and sorted into Group A and Group B, according to similarities in the composition. They were submitted to the chemical analysis of nutrients and minerals. Results were compared with the nutritional requirements proposed by the Reference Dietary Recommendation to men aged 51-70 years. Results and discussion: The analysis showed diets with a normal distribution of protein, lipids and carbohydrates. The total amount of dietary fiber in the diet of Group A was between $12 \mathrm{~g}$ and $15 \mathrm{~g}$ with $48 \%$ of soluble fiber. In Group B dietary fiber was $3.40 \mathrm{~g}$, and in the standard lactose-free diet, between 6 and $8 \mathrm{~g}$, with $65 \%$ of soluble fiber. Concerning minerals, all formulations were appropriate in iron and most of them in calcium, zinc, phosphorus, copper and sodium. No formulation evaluated presented appropriate values of potassium and magnesium. We observed low adequacy of many minerals in the formulations of $1.200 \mathrm{Kcal}$. Conclusion: The formulations analyzed have adequate macronutrients, but require adjustments in fiber. Minerals in reduced amounts need to be supplemented while dietary adjustments are not made.

Key words: Food Security and Nutrition. Homecare. Enteral Nutrition. Macronutrients. Chemical Composition. Health System. Adults.

\section{Introdução}

A ampliação do atendimento em domicílio a pessoas que precisam de cuidados regulares ou intensivos, mas não de hospitalização, é uma das estratégias contempladas no Plano de Ações para Enfrentamento das Doenças Crônicas Não Transmissíveis (DCNT) do Ministério da Saúde, ${ }^{1}$ objetivando o cuidado integral e a promoção da saúde. Nesse contexto, a terapia nutricional enteral (TNE) no domicílio tem sido cada vez mais indicada, por ser alternativa viável e vantajosa, promovendo o convívio dos pacientes com seus familiares, além de melhorar o conforto e a qualidade de vida, ${ }^{2,3}$ contribuindo para a garantia do direito à alimentação adequada e saudável aos portadores de necessidades alimentares especiais. ${ }^{4-6}$ 
Nutrição enteral é definida pela Agência Nacional de Vigilância Sanitária como todo alimento para fins especiais, formulado e elaborado para uso em cateteres ou via oral, podendo ser industrializada ou não. ${ }^{7}$ No Brasil, o uso de dietas artesanais e/ou semiartesanais é incentivado para indivíduos sob cuidados no domicílio. ${ }^{8}$

As fórmulas artesanais ou semiartesanais são constituídas por alimentos in natura ou/e produtos alimentícios e, ainda, módulos de nutrientes. ${ }^{7}$ Apresentam como vantagem o menor custo e melhoria da identidade sociocultural com o alimento ofertado, auxiliando na desmistificação da nutrição enteral como alimentação artificial $^{9}$ e reconhecendo sua existência no espaço dos hábitos de consumo alimentar. ${ }^{10}$ No entanto, apresentam desvantagens, como falta de padronização das medidas caseiras, além de muitas vezes seus componentes exigirem excessiva diluição para alcançar uma viscosidade aceitável. Os procedimentos de preparo e o tempo de cocção também podem comprometer a composição nutricional da dieta. ${ }^{11,12}$

Na literatura, são escassos os trabalhos que propõem dietas enterais artesanais completas nutricionalmente, de baixo custo, com adequada estabilidade e osmolalidade. A maioria dos estudos disponíveis sobre composição química das dietas enterais artesanais utiliza tabelas de composição química, sem levar em conta as perdas que ocorrem devido ao processamento dos alimentos, ${ }^{13}$ havendo poucas iniciativas de estudos bromatológicos e experimentais que quantifiquem precisamente os macro e micronutrientes. Recentemente, observa-se a estruturação de algumas propostas adequadas em macronutrientes, mas, ainda com raros estudos quanto aos micronutrientes, tendo em vista as dificuldades com os métodos analíticos e seu custo. ${ }^{12,14,15}$

Portanto, torna-se estratégico e necessário o estudo analítico e experimental da matriz alimentar que corrobore e norteie a prática dietética para que se determine com precisão e exatidão a composição de dietas artesanais tanto em macronutrientes quanto em minerais. Assim, será possível oferecer subsídios a uma proposta de dieta enteral adequada nutricionalmente, de baixo custo e de fácil preparo, reduzindo os erros provenientes de medidas caseiras e minimizando os erros de protocolos de preparo advindos do exercício da técnica dietética.

Nessa perspectiva, o objetivo deste estudo é avaliar quimicamente a composição de macronutrientes e minerais de dietas artesanais prescritas na alta hospitalar de instituições de referência do Estado de Minas Gerais, possibilitando a criação de uma referência analítica para a manipulação e administração corretas de nutrientes pela via enteral. 


\section{Materiais e métodos}

As fichas técnicas das dietas enterais artesanais padrão e padrão sem lactose, prescritas na alta hospitalar por oito hospitais públicos do município de Belo Horizonte-MG e pelo Núcleo de Apoio à Saúde da Família (NASF) da Secretaria Municipal de Saúde, foram coletadas junto aos nutricionistas responsáveis pela dispensação das prescrições. Considerou-se dieta padrão a dieta normocalórica e normoproteica, sem restrição de nutrientes, com exceção da lactose nas formulações isenta de lactose.

Todos os valores de medidas caseiras ou medidas padronizadas pelo serviço foram equalizados e convertidos para medidas de massa, permitindo a correta transposição dos dados informados. Em seguida, as dietas foram preparadas em Laboratório de Técnica Dietética e avaliadas quanto a viscosidade, estabilidade, odor, cor e custo, sendo incluídas no estudo somente as dietas aprovadas em todos estes quesitos.

O teste de viscosidade foi realizado administrando-se as dietas por cateteres nasogástricas e nasoentéricas para indivíduos adultos, calibre 12, pelo método gravitacional, com auxílio de equipo para catéter e em bolus por meio de seringas de $20 \mathrm{ml}$. Considerou-se com adequada viscosidade a administração das dietas por ambos os métodos sem entupimento do catéter. A estabilidade das dietas foi testada por inspeção visual do processo de separação de fases em período de 12 horas de armazenamento refrigerado. Inspeção olfativa e visual avaliaram o odor e a cor. Todas apresentaram odor e cor agradáveis. Foram considerados como de custo aceitável os valores diários máximos de 12 reais, ou meio salário mínimo por mês em ofertas diárias de 1.800 quilocalorias.

Após estas análises, foram aprovadas as fichas técnicas de cinco locais. As fichas dos demais locais foram eliminadas do estudo por apresentarem custo elevado $(n=1)$ ou viscosidade inadequada $(n=3)$. Assim, foram incluídas no estudo as formulações de dietas padrão e padrão sem lactose (quadros 1 e 2), cada uma com dois níveis calóricos de maior emprego na prática clínica, segundo informações colhidas junto aos serviços de nutrição dos locais selecionados.

Três locais, denominados Grupo A, utilizavam a mesma dieta padrão, sendo apresentadas neste trabalho as formulações de 1.800 e 2.100 quilocalorias (Kcal). Os outros dois locais, denominados Grupo B, utilizavam dietas semelhantes, mas além da padrão, prescreviam também a padrão sem lactose. Neste estudo analisamos as formulações de 1.200 e 2.100 Kcal padrão e de 1.200 e 1.800 Kcal sem lactose (quadros 1 e 2). A não padronização do valor calórico entre os grupos ocorreu em decorrência de diferenças nas prescrições pelos nutricionistas responsáveis. Amostras de 100 $\mathrm{ml}$ foram encaminhadas para análise química, conforme descrito a seguir. 
Quadro 1. Formulações e características das dietas artesanais enterais padrão, prescritas em altas hospitalares por dois hospitais públicos e pelo Núcleo de Apoio à Saúde da Família (NASF) da Secretaria Municipal de Saúde, Belo Horizonte, março de 2014.

\begin{tabular}{|c|c|c|}
\hline Alimentos & Padrão $1.800 \mathrm{Kcal}$ & Padrão $2.100 \mathrm{Kcal}$ \\
\hline Leite integral (ml) & 500,00 & 500,00 \\
\hline Leite desnatado $(\mathrm{ml})$ & 500,00 & 500,00 \\
\hline Albumina em pó (g) & 11,60 & $\begin{array}{c}29,00 \\
23,20(2 x / \text { semana })\end{array}$ \\
\hline Farinha de trigo torrada $(\mathrm{g})$ & 117,00 & 117,00 \\
\hline Batata cozida $(\mathrm{g})$ & 280,00 & 280,00 \\
\hline Castanha do Pará (g) & 2,00 & 2,00 \\
\hline Óleo de soja (g) & 39,00 & 52,00 \\
\hline Cereal comercial a base de milho $(\mathrm{g}) *$ & 15,00 & 20,00 \\
\hline Açúcar (g) & 27,60 & 27,50 \\
\hline Sal iodado $(\mathrm{g})$ & 2,00 & 4,00 \\
\hline Ovo cozido $(\mathrm{g})$ & $45,00(2 \mathrm{x} /$ semana $)$ & $45,00(2 x /$ semana $)$ \\
\hline Maltodextrina (g) & ---- & ---- \\
\hline \multicolumn{3}{|l|}{ Suco $(250 \mathrm{ml})$} \\
\hline Cenoura crua $(\mathrm{g})$ & 55,00 & 55,00 \\
\hline Suco de laranja (ml) & 180,00 & 180,00 \\
\hline Açúcar & 13,80 & 27,50 \\
\hline Maltodextrina & ---- & ---- \\
\hline Volume final sem suco (ml) & 1625 & 1900 \\
\hline Volume final com suco (ml) & 1875 & 2150 \\
\hline Distribuição & $5 \times 325 \mathrm{ml}+$ suco & $5 \times 380 \mathrm{ml}+$ suco \\
\hline Custo por dia $(\mathrm{R} \$) * *$ & 5,57 & 6,68 \\
\hline
\end{tabular}

*Ingredientes do cereal comercial à base de milho enriquecido: farinha de milho, açúcar, enriquecido com ferro, fósforo, cálcio zinco, ácido fólico, niacina, vitaminas A, D, E, C, B1 e B6.

** Cotação realizada em março de 2014, no comércio varejista de Belo Horizonte. 
Quadro 2. Formulações e características das dietas artesanais enterais padrão e padrão sem lactose, prescritas em altas hospitalares por dois hospitais públicos. Belo Horizonte, março de 2014

\begin{tabular}{|c|c|c|c|c|}
\hline Alimentos & $\begin{array}{c}\text { Padrão } \\
1.200 \\
\text { Kcal }\end{array}$ & $\begin{array}{c}\text { Padrão } \\
2.100 \\
\text { Kcal }\end{array}$ & $\begin{array}{c}\text { Sem } \\
\text { Lactose } \\
1.200 \mathrm{Kcal}\end{array}$ & $\begin{array}{c}\text { Sem } \\
\text { Lactose } \\
1.800 \mathrm{Kcal}\end{array}$ \\
\hline Leite em pó integral (g) & 110,0 & 195,0 & ---- & ---- \\
\hline $\begin{array}{l}\text { Complemento alimentar a base de } \\
\text { extrato de soja enriquecido }(\mathrm{g}) *\end{array}$ & 105,0 & 180,0 & ---- & ---- \\
\hline $\begin{array}{l}\text { Cereal comercial a base de } \\
\text { milho enriquecido }(\mathrm{g}) * *\end{array}$ & 63,0 & 117,0 & ---- & ---- \\
\hline Sal iodado $(\mathrm{g})$ & 2,0 & 2,0 & 2,0 & 2,0 \\
\hline Óleo de soja (g) & ---- & ---- & 5,0 & 5,0 \\
\hline Leite de soja em pó (g) & ---- & ---- & 150,0 & 215,0 \\
\hline Creme de arroz enriquecido $(\mathrm{g})$ & ---- & ---- & 140,0 & 140,0 \\
\hline Amido de milho (g) & ---- & ---- & 125,0 & 100,0 \\
\hline \multicolumn{5}{|l|}{ Suco $(200 \mathrm{ml})$} \\
\hline Suco de laranja (ml) & 120,0 & 120,0 & 120,0 & 120,0 \\
\hline Cenoura crua $(\mathrm{g})$ & 55,0 & 55,0 & 55,0 & 55,0 \\
\hline Volume final sem suco $(\mathrm{ml})$ & 1.200 & 1.800 & 1.200 & 1.800 \\
\hline Volume final com suco (ml) & 1400 & 2000 & 1400 & 2000 \\
\hline Distribuição & $\begin{array}{l}6 \times 200 \mathrm{ml} \\
+ \text { suco }\end{array}$ & $\begin{array}{c}6 \times 300 \mathrm{ml}+ \\
\text { suco }\end{array}$ & $\begin{array}{l}6 \times 200 \mathrm{ml} \\
+ \text { suco }\end{array}$ & $\begin{array}{c}6 \times 300 \mathrm{ml}+ \\
\text { suco }\end{array}$ \\
\hline Custo por dia $(\mathrm{R} \$) * * *$ & 5,96 & 10,05 & 10,47 & 12,92 \\
\hline
\end{tabular}

* Ingredientes do complemento alimentar à base de extrato de soja enriquecido: açúcar, extrato de soja, farinha de arroz, soro de leite, maltodextrina, sal refinado, óleo de soja, enriquecido com vitaminas C, B1, B2, B6, A e niacina.

** Ingredientes do cereal comercial à base de milho enriquecido: farinha de milho, açúcar, enriquecido com ferro, fósforo, cálcio zinco, ácido fólico, niacina, vitaminas A, D, E, C, B1 e B6.

*** Cotação realizada em março de 2014, no comercio varejista de Belo Horizonte. 
Tanto os macronutrientes quanto os micronutrientes foram analisados em triplicatas. Foram adotados os métodos padrão recomendados pela Association of Analytical Chemists (AOAC) ${ }^{16}$ A proteína foi quantificada pelo método de semimicro KJeldahl, sendo a conversão do nitrogênio total em proteína bruta pelo fator $6,25 .{ }^{16}$ Os lipídios totais foram estimados segundo procedimento descrito por Bligh \& Dyer ${ }^{17}$ (homogeneizador, AP-22, Tecnal ${ }^{\circledR}$, Piracicaba, Brasil). A fibra alimentar solúvel e insolúvel, pelo método enzimático ${ }^{18}$ (banho metabólico com agitação reciprocante, tipo Dubnoff, MA-093, Marconi ${ }^{\circledR}$, Piracicaba, Brasil) e teor de cinzas, pelo método de incineração em mufla (EDGCON 1P, EDG Equipamentos ${ }^{\circledR}$, São Paulo, Brasil) a $550^{\circ} \mathrm{C}$ e de umidade, amostras integrais, em estufa (M-315 SE, Fanem ${ }^{\circledR}$, São Paulo, Brasil) $105^{\circ} \mathrm{C}$, até peso constante. Os carboidratos totais foram estimados por diferença, diminuindo-se de 100 a somatória de proteínas, lipídios, cinzas, umidade e fibra alimentar solúvel e insolúvel. ${ }^{16}$ Os resultados foram expressos em $\mathrm{g} / 100 \mathrm{~g}$ de dieta.

A energia metabolizável foi calculada utilizando-se os fatores de conversão de 4 kcal para carboidrato e proteína e de 9 kcal para lipídios. Foram também quantificados os minerais zinco $(\mathrm{Zn})$, ferro $(\mathrm{Fe})$, cobre $(\mathrm{Cu})$, cálcio $(\mathrm{Ca})$, fósforo $(\mathrm{P})$, potássio $(\mathrm{K})$, magnésio $(\mathrm{Mg})$, manganês (Mn) e selênio (Se) em equipamento de ICP-OES, marca Varian (720 ICP-OES, Varian Inc., Califórnia, US), utilizando as seguintes condições experimentais: Potência: 1,20 KW, Fluxo de plasma: $15.0 \mathrm{~L} / \mathrm{min}$, Fluxo de gás auxiliar: $1.50 \mathrm{~L} / \mathrm{min}$, Pressão do nebulizador: $200 \mathrm{Kpa}$, utilizando-se respectivamente as seguintes linhas espectrais: 206,2 nm, 238,2 nm, 327,4 nm, $317,9 \mathrm{~nm}, 213,6 \mathrm{~nm}, 766,4 \mathrm{~nm}, 285,2$ e 257,6. A escolha das linhas espectrais analíticas foi baseada na sensibilidade e nível de interferência. Intervalos de concentração lineares para cada elemento variaram entre seu limite de detecção e os valores máximos de concentração recomendados pelos manuais do fabricante. Os limites de detecção (3 x o desvio-padrão de 10 medidas do branco analítico dividido pelo pela inclinação da curva de calibração) foram determinados para todos os elementos lidos. 
Para a leitura das amostras de selênio (Se), foi utilizado um sistema de gerador de hidretos $\left(\mathrm{NABH}_{4}\right)$, montado sobre uma plataforma V-groove com nebulizador do tipo Babington, com orifício de gás de 70 mm acoplado. Soluções estoque de $1000 \mu \mathrm{g} \cdot \mathrm{mL}^{-1}$ (Spex Sample Preparation, Metuchen, NJ) dos elementos foram utilizadas para preparar as curvas de calibração e otimização das condições analíticas. Todos os padrões foram preparados da mesma forma que as amostras. O Se (VI) foi reduzido para Se (IV) em $\mathrm{HCl} 4 \mathrm{M}$, esquentando a solução a $90^{\circ} \mathrm{C}$ por $30 \mathrm{~min}$. Todas as soluções aquosas, incluindo-se as diluições para a leitura das amostras de dietas, foram preparadas com água ultrapura $\left(18 \mathrm{M} \Omega \mathrm{cm}^{-1}\right)$, Milli- $Q$ (Millipore, Bedford, MA). Material de referência certificado - NIST - Total Diet SRM 1548 (National Institute of Standards Technology - Gaithersburg, MD) foi determinado para validar as medidas analíticas obtidas no espectrômetro de ICP-OES.

Após a análise, os resultados obtidos foram comparados com as necessidades nutricionais propostas pelas Recomendações Dietéticas de Referência (DRIs) para homens saudáveis considerando a faixa etária de 51-70 anos. ${ }^{19,20}$ Optou-se por esta faixa etária e sexo, pois a maioria dos pacientes em uso de TNE no domicílio têm idade superior a 50 anos e as recomendações de minerais para o sexo masculino são maiores do que as do feminino, cobrindo, assim, as necessidades de ambos, exceto para o cálcio. A adequação dos minerais ferro, cobre, zinco, cálcio, fósforo, magnésio e selênio foi realizada comparando-se o valor obtido na análise com a Ingestão Dietética Recomendada (RDA) e com o Nível Máximo Tolerável de Ingestão (UL) considerando-se oferta adequada valores entre a RDA e a UL. O sódio, o potássio e o manganês, que não têm RDA estabelecida, foram avaliados em relação à Ingestão Adequada (AI) e ao UL. ${ }^{19,20}$ Adicionalmente, o sódio e o potássio foram analisados quanto a sua relação (sódio/potássio), com enfoque no controle da pressão arterial e saúde cardiovascular, considerando-se adequados valores entre 0,49 e 0,32.19, 21

Apesar de este estudo não ter como foco a qualidade microbiológica das dietas, o preparo das mesmas foi realizado considerando critérios de manipulação segura. Os mesmos cuidados são orientados aos cuidadores do paciente na alta hospitalar e são checados in loco pelos nutricionistas do NASF na visita domiciliar.

Todos os hospitais participantes, assim como o NASF, são parceiros do projeto no qual este estudo está inserido e assinaram termo de compromisso, concordando na disponibilização das fórmulas de dietas enterais artesanais orientadas na alta hospitalar para análise química e divulgação dos resultados a cega. 


\section{Resultados}

Observando-se os dados de composição centesimal, base úmida (tabela 1), a distribuição dos macronutrientes, proteínas, carboidratos e lipídeos, em relação às calorias, foi de aproximadamente 14,0\%, 50,0\% e 36,0\% nas formulações padrão do Grupo A, e de 16,5\%, $49,0 \%$ e 34,5\% nas formulações padrão do Grupo B, respectivamente. As formulações sem lactose apresentaram distribuição para proteínas, carboidratos e lipídeos de aproximadamente $17,0 \%, 56,0 \%$ e $27,0 \%$.

Quanto à umidade (tabela 1), o valor médio para as dietas de ambos os grupos foi de 78\%, sem considerar o suco. Valores abaixo de $80 \%$ são considerados baixos e, conforme encontrado nas análises deste estudo, denotam a grande quantidade de solutos empregados para a formulação de todas as dietas em tela, independentemente do grupo a que pertencem.

A quantidade total de fibra alimentar nas dietas do Grupo A foi de 11,86 g para o padrão 1.800 Kcal e de 15,46 g para o padrão $2.100 \mathrm{Kcal}$, com $48 \%$ de fibra solúvel em ambas. Isso não ocorre nas dietas padrão do Grupo B, cujo aporte de fibra alimentar é exclusivamente em virtude do suco, sendo de 3,40 g para ambos os padrões calóricos. Na formulação isenta de lactose o teor é um pouco mais elevado, com 6,04 e 8,44 g para as dietas de 1.200 e $1.800 \mathrm{Kcal}$, respectivamente, apresentando aproximadamente $65 \%$ de fibra solúvel.

No tocante ao aporte calórico total ofertado pelas dietas analisadas (dieta + suco), as do Grupo A apresentam um total de calorias compatível com os níveis calóricos planejados e calculados por dados de tabelas de composição centesimal de alimentos, não excedendo mais do que $5,5 \%$ da meta calórica pretendida. O mesmo comportamento analítico pode ser observado na dieta padrão $1.200 \mathrm{Kcal}$ e nas dietas padrão sem lactose do Grupo B, evidenciando que, apesar das diferenças na composição e na fonte primária de nutrientes entre as dietas dos diferentes grupos, todas elas atendem corretamente à demanda de macronutrientes para a qual foram propostas. No entanto, a dieta padrão $2.100 \mathrm{Kcal}$ do Grupo B apresenta 2.417 Kcal, ou $15 \%$ acima do planejado. 
Tabela 1. Composição centesimal (\% base úmida) das formulações enterais artesanais prescritas em altas hospitalares de quatro diferentes hospitais públicos e Núcleo de Apoio à Saúde da Família (NASF) da Secretaria Municipal de Saúde. Belo Horizonte, março de 2014

FORMULAÇÕES

Grupo A

Grupo B

\begin{tabular}{|c|c|c|c|c|c|c|c|c|}
\hline & $\begin{array}{c}\text { Padrão } \\
1.800 \\
\text { Kcal }\end{array}$ & $\begin{array}{c}\text { Padrão } \\
2.100 \\
\text { Kcal }\end{array}$ & Suco & $\begin{array}{c}\text { Padrão } \\
1.200 \\
\text { Kcal }\end{array}$ & $\begin{array}{c}\text { Padrão } \\
2.100 \\
\text { Kcal }\end{array}$ & $\begin{array}{c}\mathrm{SL}^{*} \\
1.200 \\
\text { Kcal }\end{array}$ & $\begin{array}{c}\text { SL } \\
1.800 \\
\text { Kcal }\end{array}$ & Suco \\
\hline Umidade & 77,99 & 77,32 & 81,47 & 80,38 & 77,03 & 78,81 & 78,06 & 86,05 \\
\hline $\begin{array}{l}\text { Proteína } \\
\text { bruta (N x } \\
6,25)\end{array}$ & 3,67 & 3,71 & 1,27 & 3,95 & 4,53 & 4,03 & 4,28 & 1,11 \\
\hline $\begin{array}{l}\text { Lipídios } \\
\text { Totais }\end{array}$ & 4,31 & 4,34 & 0,15 & 3,75 & 4,23 & 2,90 & 3,09 & 0,13 \\
\hline Carboidratos & 13,08 & 13,52 & 14,55 & 11,46 & 13,59 & 13,51 & 13,75 & 10,59 \\
\hline $\begin{array}{l}\text { Fibra } \\
\text { Alimentar } \\
\text { Solúvel }\end{array}$ & 0,12 & 0,16 & 1,50 & 0,00 & 0,00 & 0,12 & 0,16 & 1,29 \\
\hline $\begin{array}{l}\text { Fibra } \\
\text { Alimentar } \\
\text { Insolúvel }\end{array}$ & 0,29 & 0,38 & 0,58 & 0,00 & 0,00 & 0,10 & 0,12 & 0,41 \\
\hline $\begin{array}{l}\text { Fibra } \\
\text { Alimentar } \\
\text { Total }\end{array}$ & 0,41 & 0,54 & 2,08 & 0,00 & 0,00 & 0,22 & 0,28 & 1,70 \\
\hline Cinzas & 0,54 & 0,58 & 0,48 & 0,46 & 0,62 & 0,53 & 0,54 & 0,42 \\
\hline $\begin{array}{l}\text { Calorias } \\
\text { (Kcal) }\end{array}$ & 105,79 & 107,98 & 64,63 & 95,39 & 110,55 & 96,26 & 100,01 & 47,97 \\
\hline
\end{tabular}

$* \mathrm{SL}=$ Sem Lactose

A tabela 2 apresenta os valores médios e respectivos desvios padrão dos minerais analisados. Observa-se que, em geral, o aporte de ferro, cobre, zinco e cálcio é mais elevado no Grupo B. Maiores aportes de selênio e potássio são observados nas formulações do grupo A. No entanto, o potássio está abaixo da AI e sua relação com sódio também está inadequada em todas as 
formulações. As dietas padrão sem lactose estão provavelmente com deficiência de manganês. Nenhuma formulação analisada está adequada em magnésio. Sódio está abaixo da AI somente nas dietas padrão sem lactose e acima da UL na dieta padrão de $2.100 \mathrm{Kcal}$ do Grupo A.

Tabela 2. Concentrações de minerais das formulações enterais artesanais prescritas em altas hospitalares de quatro diferentes hospitais públicos e Núcleo de Apoio à Saúde da Família (NASF) da Secretaria Municipal de Saúde. Belo Horizonte, março de 2014.

\begin{tabular}{|c|c|c|c|c|c|c|}
\hline & \multicolumn{6}{|c|}{ FORMULAÇÕES } \\
\hline & \multicolumn{2}{|c|}{$\begin{array}{c}\text { Grupo A } \\
\text { Média (DP) }\end{array}$} & \multicolumn{4}{|c|}{$\begin{array}{c}\text { Grupo B } \\
\text { Média (DP) }\end{array}$} \\
\hline & Padrão & Padrão & Padrão & Padrão & $\mathrm{SL}^{*}$ & $\mathrm{SL}$ \\
\hline & 1.800 & 2.100 & 1.200 & 2.100 & 1.200 & 1.800 \\
\hline & Kcal & Kcal & Kcal & Kcal & Kcal & Kcal \\
\hline Ferro (mg) & $\begin{array}{c}7,92 \\
(0,77)\end{array}$ & $\begin{array}{c}8,88 \\
(0,50)\end{array}$ & $\begin{array}{l}26,28 \\
(1,68)\end{array}$ & $\begin{array}{l}35,08 \\
(1,93)\end{array}$ & $\begin{array}{l}27,11 \\
(2,42)\end{array}$ & $\begin{array}{l}36,40 \\
(2,28)\end{array}$ \\
\hline Cobre (mg) & $\begin{array}{c}3,01 \\
(0,19)\end{array}$ & $\begin{array}{c}5,76 \\
(0,37)\end{array}$ & $\begin{array}{c}6,80 \\
(0,44)\end{array}$ & $\begin{array}{c}9,73 \\
(0,58)\end{array}$ & $\begin{array}{l}14,01 \\
(1,13)\end{array}$ & $\begin{array}{l}16,22 \\
(1,37)\end{array}$ \\
\hline Zinco (mg) & $\begin{array}{c}9,74 \\
(1,37)\end{array}$ & $\begin{array}{l}10,92 \\
(0,92)\end{array}$ & $\begin{array}{l}17,31 \\
(1,26)\end{array}$ & $\begin{array}{l}21,95 \\
(1,73)\end{array}$ & $\begin{array}{l}22,59 \\
(2,76)\end{array}$ & $\begin{array}{l}25,12 \\
(2,01)\end{array}$ \\
\hline Cálcio (mg) & $\begin{array}{c}1.116,59 \\
(88,21)\end{array}$ & $\begin{array}{c}1.161,63 \\
(96,13)\end{array}$ & $\begin{array}{l}2.285,02 \\
(106,92)\end{array}$ & $\begin{array}{c}2.992,79 \\
(174,85)\end{array}$ & $\begin{array}{c}1.283,66 \\
(95,74)\end{array}$ & $\begin{array}{l}1.927,84 \\
(108,14)\end{array}$ \\
\hline Fósforo (mg) & $\begin{array}{l}976,74 \\
(59,16)\end{array}$ & $\begin{array}{c}1.137,81 \\
(74,18)\end{array}$ & $\begin{array}{c}1.174,93 \\
(95,41)\end{array}$ & $\begin{array}{c}1.942,94 \\
(112,15)\end{array}$ & $\begin{array}{l}509,70 \\
(27,19)\end{array}$ & $\begin{array}{l}578,12 \\
(25,85)\end{array}$ \\
\hline Potássio (mg) & $\begin{array}{l}2.199,26 \\
(100,38)\end{array}$ & $\begin{array}{c}2.202,45 \\
(118,44)\end{array}$ & $\begin{array}{l}1.581,66 \\
(133,02)\end{array}$ & $\begin{array}{l}1962,37 \\
(125,98)\end{array}$ & $\begin{array}{l}507,03 \\
(29,06)\end{array}$ & $\begin{array}{l}726,69 \\
(33,08)\end{array}$ \\
\hline Magnésio (mg) & $\begin{array}{l}260,31 \\
(13,54)\end{array}$ & $\begin{array}{l}277,40 \\
(17,14)\end{array}$ & $\begin{array}{c}146,46 \\
(8,19)\end{array}$ & $\begin{array}{l}214,18 \\
(12,90)\end{array}$ & $\begin{array}{l}139,50 \\
(13,63)\end{array}$ & $\begin{array}{l}169,46 \\
(12,03)\end{array}$ \\
\hline Manganês (mg) & $\begin{array}{c}2,14 \\
(0,09)\end{array}$ & $\begin{array}{c}3,44 \\
(0,18)\end{array}$ & $\begin{array}{c}2,31 \\
(0,18)\end{array}$ & $\begin{array}{l}2,94 \\
(0,32)\end{array}$ & $\begin{array}{c}1,51 \\
(0,17)\end{array}$ & $\begin{array}{c}2,18 \\
(0,15)\end{array}$ \\
\hline Selênio $(\mu \mathrm{g})$ & $\begin{array}{l}127,60 \\
(11,45)\end{array}$ & $\begin{array}{l}131,52 \\
(10,63)\end{array}$ & $\begin{array}{l}12,05 \\
(0,89)\end{array}$ & $\begin{array}{l}13,80 \\
(1,07)\end{array}$ & $\begin{array}{l}12,66 \\
(1,45)\end{array}$ & $\begin{array}{l}14,04 \\
(1,33)\end{array}$ \\
\hline Sódio (mg) & $\begin{array}{l}1.751,17 \\
(102,89)\end{array}$ & $\begin{array}{l}2.557,91 \\
(136,79)\end{array}$ & $\begin{array}{l}1.927,36 \\
(171,07)\end{array}$ & $\begin{array}{l}2.240,52 \\
(185,29)\end{array}$ & $\begin{array}{l}1.191,89 \\
(129,94)\end{array}$ & $\begin{array}{c}1.503,24 \\
(121,81)\end{array}$ \\
\hline
\end{tabular}

${ }^{*} \mathrm{SL}=$ Sem Lactose 


\section{Discussão}

As dietas analisadas, de ambos os grupos, apresentaram composição nutricional normocalórica, normoproteica e normolipídica. Em recente trabalho, Santos e colaboradores ${ }^{12}$ encontraram $^{2}$ resultados semelhantes ao avaliarem a distribuição de macronutrientes das dietas enterais artesanais prescritas em domicílio. A exceção foram os carboidratos, cujo valor encontrado foi superior (16,9 $\mathrm{g}$ de carboidratos em $100 \mathrm{~g}$ de dieta) ao encontrado nas análises do presente estudo (em média $13 \mathrm{~g}$ de carboidrato por $100 \mathrm{~g}$ de dieta). Tal fato pode ocorrer devido a diferenças na fonte de carboidratos utilizados nas dietas.

Aquelas analisadas por Santos e colaboradores ${ }^{12}$ possuem como principal fonte a maltodextrina, enquanto as do Grupo A apresentam farinha de trigo, batata e açúcar como principais fontes de carboidrato e as dietas do Grupo B, complemento alimentar à base de extrato de soja enriquecido com vitaminas e minerais e cereal comercial à base de milho enriquecido com vitaminas e minerais como fontes de carboidrato da dieta padrão, e amido de milho e creme de arroz na dieta padrão sem lactose.

Observa-se que nas dietas há presença de sacarose, ingrediente aceitável em uma dieta padrão. Além do açúcar, o cereal comercial e o complemento de soja contêm, respectivamente, 20\% e 25\% do total de calorias compostos por sacarose (informações fornecidas pelos fabricantes). Isto resulta em 5,6\% e 8,4\% do total de calorias provindas da sacarose para as dietas padrão do Grupo A e Grupo B respectivamente, valores considerados aceitáveis..$^{19}$ As dietas padrão sem lactose não possuem sacarose na formulação.

O aporte proteico das dietas analisadas encontra-se adequado considerando-se os valores percentuais estabelecidos nas DRIs para homens adultos na faixa etária de 51 a 70 anos. ${ }^{19}$ Porém, é importante ressaltar que, quando este valor é convertido em gramas por quilo de peso, os valores das dietas de $1.200 \mathrm{Kcal}$ do grupo B (0,70 e 0,72 gramas de proteína por Kg de peso, considerando um adulto de $70 \mathrm{Kg}$ ) ficam abaixo do preconizado pelas Recomendações Nutricionais para Adultos em Terapia Nutricional Enteral e Parenteral. ${ }^{22}$ Este dado é importante, uma vez que a proteína é nutriente essencial envolvido nos processos de cicatrização e função imunológica. ${ }^{23}$ Além disso a Terapia Nutricional Enteral Domiciliar atende a um perfil variado de pacientes, geralmente adultos ou idosos, que em geral são portadores de doenças que elevam a demanda calórica e que podem cursar com alguma forma de desnutrição. ${ }^{24}$ Assim, o aporte proteico adequado deve ser sempre priorizado na elaboração das dietas enterais artesanais.

Embora a contribuição percentual de calorias provenientes dos lipídios em ambos os grupos esteja adequada, ${ }^{19}$ o perfil de ácidos graxos destas dietas, calculados por meio de tabela de composição química de alimentos (dados não apresentados), não atende plenamente às recomendações. ${ }^{19}$ As dietas do Grupo A apresentaram menores valores de ácidos graxos monoinsaturados e 
intermediário de saturado, enquanto as dietas do Grupo B apresentaram elevado teor de ácidos graxos saturados e muito baixo de monoinsaturados. Estão bem estabelecidos na literatura os efeitos protetores dos ácidos graxos monoinsaturados e polinsaturados na saúde cardiovascular e, em contrapartida, os efeitos deletérios dos ácidos graxos saturados. ${ }^{25}$

Os ingredientes das dietas originais incluíam óleo de canola (dieta do Grupo A) ou azeite de oliva (dietas do Grupo B). No entanto, os serviços de nutrição prescritores dessas dietas alteraram esta formulação para $100 \%$ de óleo de soja, em virtude da relação custo-benefício e da análise de que essa modificação não traria impacto significativo no perfil de ácidos graxos, levando-se em consideração a boa proporção entre ácidos graxos n-6 e n-9 apresentada pelo óleo de soja. Ressalta-se que, nas dietas do Grupo A, a substituição de $50 \%$ do óleo vegetal da fórmula por óleo de canola aumentaria a proporção de ácido graxo monoinsaturado, com impacto no custo mensal de 3,30 reais. A introdução de azeite de oliva virgem nas dietas padrão sem lactose do Grupo B aumentaria o custo em 3,20 reais por mês. A dieta padrão do Grupo B não tem óleo vegetal na sua composição, sendo o lipídio da dieta proveniente do leite em pó integral e do complemento alimentar à base de extrato de soja - portanto, a adequação do perfil de ácidos graxos deverá considerar mudanças mais profundas na formulação.

A quantidade de fibra alimentar nas dietas do Grupo A ficou $50 \%$ abaixo do recomendado para a faixa de homens de 51 a 70 anos, ${ }^{19}$ enquanto que, nas dietas do Grupo B, foram encontrados percentuais de adequação ainda menores (adequação média de 30\%). Destaca-se o elevado percentual de fibras solúveis, sendo em torno de $48 \%$ nas dietas do Grupo A e de $65 \%$ nas do Grupo B. O teor de fibras encontrado na dieta padrão de $2.100 \mathrm{Kcal}$ do Grupo A é maior do que em outros estudos e semelhante nas demais formulações. ${ }^{15,26,27} \mathrm{O}$ que provavelmente contribuiu para o teor de fibras das dietas analisadas foi a forma de preparo das mesmas, em que somente o suco foi peneirado, além da característica dos ingredientes in natura das formulações do Grupo A.

A fibra possui papel importante no organismo, pois está envolvida com adequado funcionamento intestinal. Ainda, a fibra solúvel é fermentada, produzindo ácidos graxos de cadeia curta envolvidos no trofismo intestinal e na manutenção da microbiota saudável..$^{28,29}$ No entanto, sabese que a adequação de fibras alimentares em dietas enterais artesanais é uma questão, pois esta interfere diretamente na viscosidade e fluidez da dieta. ${ }^{30}$ Outro inconveniente seria a intolerância gastrintestinal, podendo provocar efeitos secundários como flatulência, distensão abdominal ou mudanças na consistência das fezes ${ }^{28,31}$ e, no caso de fibra insolúvel, a possibilidade de redução da biodisponibilidade de minerais como o cálcio e o ferro. ${ }^{32}$ Recentemente, Araújo e colaboradores ${ }^{27}$ propuseram o uso de alimentos liofilizados em dietas enterais artesanais, visando a melhor adequação das fibras dietéticas. A canela em pó, acrescida em $25 \mathrm{~g} / 2.000 \mathrm{ml}$ de fórmula foi o alimento que mais contribuiu com o aumento da fibra total. ${ }^{27}$ 
O percentual de umidade encontrada foi $76 \%$ para todas as dietas analisadas, o que é baixo se comparado a outros tipos de formulações enterais artesanais (valores acima de $85 \%$ ). ${ }^{33}$ Isso denota a grande quantidade de solutos empregados para a formulação de todas as dietas objeto deste estudo, independentemente do grupo a que pertencem.

Em relação aos minerais, estes apresentaram diferença importante em entre as dietas do Grupo A e B. Tal diferença pode ser atribuída à maior oferta de produtos industrializados na dieta do Grupo B, o que também refletiu no seu custo mais elevado. Todas as formulações estavam adequadas em ferro e a maioria em cálcio, zinco, fósforo, cobre e sódio. Nenhuma formulação avaliada encontrou valores adequados de potássio e magnésio. Observa-se, ainda, baixa adequação de muitos minerais nas formulações de $1.200 \mathrm{Kcal}$, o que deve ser avaliado cautelosamente na prescrição de padrões calóricos tão baixos.

Esses achados são parcialmente distintos dos de Von Atzingen e colaboradores, que encontraram inadequação nas concentrações dos minerais, ferro, cobre, magnésio e cálcio em dietas enterais artesanais à base de hidrolisado protéico de carne. ${ }^{15}$ Os minerais são usados como cofatores por diversas enzimas em inúmeras funções celulares, sendo importantes no metabolismo corporal, além de papel como antioxidante e no sistema imune. ${ }^{34}$

As concentrações de ferro encontradas nas análises estiveram muito próximas ao recomendado para Grupo A, enquanto que, no Grupo B, se aproximaram da UL. Este mesmo padrão pode ser observado para o mineral zinco. Trata-se de minerais importantes, pois estão envolvidos no metabolismo dos eritrócitos, além de o zinco estar diretamente envolvido na replicação celular e a proliferação dos fibroblastos. ${ }^{35}$

As dietas do Grupo A e da dieta sem lactose do Grupo B apresentaram valores de cálcio aceitáveis. ${ }^{20}$ No entanto, a dieta padrão de 2.100 Kcal do Grupo B excedeu a recomendação máxima tolerada, que é de $2.500 \mathrm{mg} .{ }^{.0}$ Indivíduos acamados, condição de muitos dos indivíduos em TNED, apresentam perda óssea rápida, ${ }^{36} \mathrm{o}$ que aumenta o risco de litíase renal em função de maior excreção de calcio urinário. ${ }^{37}$ Além disso, elevado aporte de cálcio dietético pode reduzir a absorção de magnésio. ${ }^{19}$ Neste sentido, a oferta de cálcio não deve exceder a recomendação máxima tolerável.

O fósforo está deficiente apenas nas dietas sem lactose do Grupo B. Apesar de a deficiência orgânica ser rara e a biodisponibilidade deste mineral ser elevada, ${ }^{32}$ baixos níveis plasmáticos prejudicam a saúde óssea e a força muscular, e aumentam a debilidade do paciente. ${ }^{19}$

O magnésio esteve abaixo dos valores de RDA para todas as dietas analisadas; entretanto, é descrito na literatura que a ingestão de magnésio tende a ser menor do que o recomendado mesmo nas dietas por via oral. Vários fatores estão envolvidos na diminuição da oferta de magnésio, como 
a perda durante o processo de cocção e durante a refinação dos alimentos. O magnésio é um mineral intracelular, importante para os processos fisiológicos, incluindo a função neuromuscular e manutenção de tônus cardiovascular. ${ }^{38}$ Assim, propostas de inclusão de alimentos fonte de magnesio como vegetais de cor verde escura ou mesmo desidratados devem ser avaliadas e implementadas.

A presença de dois gramas de castanha do Pará nas formulações do Grupo A foi responsável pela adequação de selênio, o que não foi encontrado nas formulações do Grupo B. Sabe-se que o selênio é incorporado nas selenoproteínas, exercendo importante papel antioxidante e anti-inflamatório. Baixos níveis de selênio têm sido associados com aumento do risco de mortalidade, alteração da função imunológica e declínio cognitivo. ${ }^{39}$

Sódio é um mineral que dispõe de vasta literatura científica com fortes evidências relacionando o baixo consumo a menores valores de pressão sistólica e diastólica em normo e hipertensos. ${ }^{40,41}$ No entanto, não há uma recomendação exata determinada. Enquanto os guias de recomendações de nutrientes orientam um consumo de 1.200 a $2.300 \mathrm{mg} /$ dia para prevenção e controle da pressão arterial $^{19}$, estudos demonstram baixo impacto na morbidade e mortalidade cardiovascular em dietas abaixo de $2.300 \mathrm{mg}{ }^{40}$ sugerindo um consumo de $3.000 \mathrm{mg} /$ dia para população em geral. ${ }^{41}$ Por outro lado, idosos, que constituem a maioria população-alvo das dietas aqui estudadas, são, do ponto de vista da homeostase hidroeletrolítica, mais responsivos à redução de sódio na dieta. ${ }^{40}$

Outro aspecto a ser considerado é a relação entre sódio e potássio. ${ }^{42}$ Estudos demonstram que uma elevada relação $\mathrm{Na} / \mathrm{K}$ tem associação independente com maior risco de doença cardiovascular e todas as causas de mortalidade, ${ }^{43}$ apontando para recomendações dietéticas de uma relação $\mathrm{Na} / \mathrm{K}$ de $0,49 .{ }^{21}$ Neste sentido, as formulações analisadas estão adequadas em sódio, exceto a dieta padrão de 2.100 do Grupo A, mas nenhuma formulação apresenta adequada relação $\mathrm{Na} / \mathrm{K}$, sendo o valor mínimo encontrado na dieta padrão de 1.800 Kcal do Grupo A $(0,80)$ e o valor máximo, na dieta sem lactose de $1.200 \mathrm{Kcal}$ do Grupo B $(2,35)$.

O cobre apresentou valores muito baixos nas dietas do Grupo A. Apesar de a deficiência deste mineral ser de baixa prevalência, ela está relacionada com anemia normocítica e hipocrômica, leucopenia e neutroponia em adultos. ${ }^{19}$

\section{Conclusão}

Os dados aqui descritos permitiram uma análise inédita da composição centesimal e de minerais de dietas artesanais empregadas na alta hospitalar de quatro hospitais de referência no Sistema Único de Saúde de Belo Horizonte e pela Atenção Básica do município. Foi possível concluir que a distribuição de macronutrientes, em geral, mostrou-se correta, havendo necessidade de adequação calórica somente em uma das 
formulações estudadas. A fibra alimentar foi encontrada em baixas concentrações em todas as dietas analisadas e deverá ser alvo de modificações que proporcionem melhor perfil desse nutriente, tanto no componente solúvel quanto no insolúvel.

$\mathrm{Na}$ análise dos minerais, foi possível verificar a necessidade de adequação por suplementação de magnésio e potássio em todas as dietas estudadas, enquanto adequações dietéticas não forem realizadas. Essa mesma recomendação é válida para o cobre nas dietas do Grupo A. Cálcio e sódio precisam ser reduzidos em duas dietas estudadas, por meio de alteração nos componentes da dieta.

Apesar da especificidade da correção dos minerais, foi possível observar que a inclusão de matrizes alimentares diversificadas, como por exemplo, castanhas ou oleaginosas, que podem ser facilmente transformadas em pó e dissolvidas, pode constituir uma via para a oferta de micronutrientes sem aumento expressivo do custo final. Estratégias como essa, que fazem parte do exercício da técnica dietética, contribuem para a melhora do perfil da oferta de nutrientes por via enteral no domicílio, aproximando a dieta dos pacientes do consumo de alimentos da família.

Ao receberem os dados aqui gerados, os hospitais e as equipes do NASF têm a oportunidade de promover as adequações necessárias às suas dietas, gerando impacto positivo na atenção nutricional e melhorando a qualidade da Terapia Nutricional Enteral Domiciliar, baseada nos preceitos da Política Nacional de Alimentação e Nutrição e previstas nas Diretrizes do Sistema Único de Saúde. Todos os resultados apresentados neste manuscrito já foram repassados aos hospitais e NASF envolvidos no estudo, que estão adequando suas dietas e suas prescrições.

\section{Agradecimentos}

Agradecemos à Fundação de Amparo à Pesquisa de Minas Gerais (FAPEMIG) e ao Ministério da Saúde, pelo apoio financeiro para a realização deste estudo. Agradecemos também a todos os parceiros não autores ou financiadores deste projeto - Serviço de Nutrição e Dietética do Hospital das Clínicas da Universidade Federal de Minas Gerais; Serviço de Nutrição e Dietética do Hospital Risoleta Tolentino Neves; Fundação Hospitalar de Minas Gerais (FHEMIG); Hospital Municipal Odilon Behrens, Instituto de Previdência e Saúde do Estado de Minas Gerais (IPSEMG); Secretaria Municipal de Saúde de Belo Horizonte e Núcleo de Apoio à Saúde da Família -, pela parceria, formatação e impressão das cartilhas. 


\section{Referências}

1. Brasil. Ministério da Saúde. Plano de ações estratégicas para enfrentamento das DCNT no Brasil 2011-2022. [Internet]. Brasília: Ministério da Saúde; 2011. [acesso em 10 mar. 2014]. Disponível em: http://portal.saude.gov.br/portal/saude/profissional/area.cfm?id_area=1818

2. Planas M, Pérez-Portabella C, Rodríguez C, Puiggrós D,. Elvira D, Dalmau E. Evaluación del grado de satisfacción de un programa de nutrición enteral domiciliaria. Nutrición Hospitalaria 2007; 22(5):612-15.

3. Cabrit R. Lambert T, Simard M, Cavaye M, Creste M, Dumery S, et al. Nutrition entérale à domicile: 3 millions de journées d'expérience. Nutrition Clinique et Métabolisme 2013; 27(4):178-184.

4. Brasil. Ministério da Saúde. Política Nacional de Alimentação e Nutrição. Brasília: Ministério da Saúde; 2012.

5. Câmara Interministerial de Segurança Alimentar e Nutricional. Plano Nacional de Segurança Alimentar e Nutricional: 2012/2015. Brasília: MDS; CAISAN; 2011.

6. Brasil. Agência Nacional de Vigilância Sanitária. Resolução de Diretoria Colegiada, RDC n 11, de 26 de janeiro de 2006. Dispõe sobre o Regulamento Técnico de Funcionamento de Serviços que prestam Atenção Domiciliar [Internet]. Diário Oficial da União 30 jan. 2006. [acesso em 20 mar. 2014]. Disponível em URL:<http://bvsms.saude.gov.br/bvs/saudelegis/anvisa/2006/ res0011_26_01_2006.html

7. Brasil. Ministério da Saúde. Resolução RDC no 63, de 6 de julho de 2000. Aprova regulamento técnico para fixar os requerimentos mínimos exigidos para a terapia nutricional enteral. Diário Oficial, 07 jul. 2000.

8. Brasil. Ministério da Saúde. Portaria 120 de 14 de abril de 2009 [Internet]. [acesso em 10 mar. 2014]. Disponível em: URL:<http://bvsms.saude.gov.br/bvs/saudelegis/sas/2009/prt0120_14_04_2009. html

9. Barbosa JAG, Freitas MIF. Representações sociais sobre a alimentação por sonda obtidas de pacientes adultos hospitalizados. Revista Latino Americana de Enfermagem 2005; 13(2):235-42.

10. Poulain JP, Proença RPC. O espaço social alimentar: um instrumento para o estudo dos modelos alimentares. Revista de Nutrição 2003; 16(3):245-256.

11. Menegassi B, Santana LS, Coelho JC, Martins AO, Pinto JPAN, Costa TMB, et al. Características físico químicas e qualidade nutricional de dietas enterais não-industrializadas. Alimentos e Nutrição. 2007; 18(2):127-132.

12. Santos VFN, Bottoni A, Morais TB. Qualidade nutricional e microbiológica de dietas enterais artesanais padronizadas preparadas nas residências de pacientes em terapia nutricional domiciliar. Revista de Nutrição 2013; 26 (2):205-14.

13. Henriques GS, Rosado GP. Formulação de dietas enterais artesanais e determinação da osmolalidade pelo método crioscópico. Revista de Nutrição 1999; 12(3):225-32. 
14. Sousa LRM, Ferreira SMR, Schieferdecker MEM. Physicochemical and nutricional characteristics of handmade enteral diets. Nutrición Hospitalaria 2014; 29:568-574.

15. Von Atzingen MC, Gabelotti ML, Araújo RFC, Soares RM, Silva MEMP. Composição centesimal e teor de minerais de dietas enterais artesanais. Revista Brasileira de Tecnologia Agroindustrial 2007; $1(2): 37-47$.

16. Association of Official Analytical Chemists. Official methods of analysis. 19. ed. Washington DC: 2012, 1141p.

17. Bligh EG, Dyer WJ. A rapid method of total lipid extraction and purification. Canadian Journal of Biochemistry and Physiology 1959; 37:911-917.

18. Asp NG, Johansson CG, Hallmer H, Siljeström M. Rapid enzymatic assay of insoluble and soluble dietary fiber. Journal of Agricultural Food Chemistry 1983; 31(3): 476-482.

19. Institute of Medicine. Dietary reference intakes. The essential guide to nutrient requirements. Washington DC: The National Academies Press; 2006. 543p.

20. Institute of Medicine. Dietary reference intake for calcium and vitamin D [Internet]. Washington D.C.: National Academies Press; 2011. [acesso em: 07 nov. 2013]. Disponível em: URL:<http://www. iom.edu/vitamind

21. U.S. Department of Agriculture. Dietary guidelines for Americans [Internet]. Washington, D.C.: USDA/DHHS; 2010. [acesso em 07 nov. 2013]. Disponível em: http://www.cnpp.usda.gov/ Publications/DietaryGuidelines/2010/PolicyDoc/PolicyDoc.pdf.

22. Sociedade Brasileira de Nutrição Parenteral e Enteral, Sociedade, Brasileira de Clínica Médica, Associação Brasileira de Nutrologia. Projeto diretrizes: recomendações nutricionais para adultos em terapia nutricional enteral e parenteral [Internet]. 2011. [acesso em 04 abr. 2014. Disponível em: http:// www.projetodiretrizes.org.br/9_volume/recomendacoes_nutricionais_de_adultos_em_terapia_ nutricional_enteral_e_parenteral.pdf

23. Crowe TC, Brockbank CM. Nutrition therapy in the prevention and treatment of pressure ulcers. Wound Practice and Research. 2009; 17:90-99.

24. Arvanitakis M, Beck A, Coppens P, De Man F, Elia M, Hebuterne X, et al. Nutrition in care homes and home care: how to implement adequate strategies. Clinical Nutrition 2008; 27(4):481-488.

25. Santos RD, Gagliardi ACM, Xavier HT, Magnoni CD, Cassani R, Lottenberg AM, et al. Sociedade Brasileira de Cardiologia. I Diretriz sobre o consumo de gorduras e saúde cardiovascular. Arquivos Brasileiros de Cardiologia. 2013; 100(1Supl.3):1-40.

26. Araújo EM, Menezes HC. Formulações com alimentos convencionais para nutrição enteral ou oral. Ciência e Tecnologia de Alimentos 2006; 26(3):533-538.

27. Araújo EM, Menezes HC, Tomazini JM. Fibras solúveis e insolúveis de verduras, tubérculos e canela para uso em nutrição clínica. Ciência e Tecnologia de Alimentos 2009; 29(2):401-406.

28. Bosaeus I. Fiber effects on intestinal functions (diarrhea, constipation and irritable bowel syndrome). Clinical Nutrition Supplements 2004; 1:33-38. 
29. Goldsmith JR, Sartor RB. The role of diet on intestinal microbiota metabolism: downstream impacts on host immune function and health, and therapeutic implications. Journal of Gastroenterology 2014; 49(5):785-798.

30. Gómez Candela C, Cos Blanco I, Iglesias Rosado C. Fibra y nutrición enteral. Nutrición Hospitalaria 2002; 17(Supl. 2):30-40.

31. Silk DB, Walters ER, Duncan HD, Green CJ. The effect of a polymeric enteral formula supplemented with a mixture of six fibers on normal human bowel function and colonic motility. Clinical Nutrition 2001; 20(1):49-58.

32. SMF. Biodisponibilidade de nutrientes. Barueri, SP: Manole: 2005. 878p.

33. Araújo EM, Menezes HC. Composição centesimal, lisina disponível e digestibilidade in vitro de proteínas de fórmulas para nutrição oral ou enteral. Ciência e Tecnologia de Alimentos 2005; 25(4):768-771.

34. Ames BN, Atamna H, Killilea DW. Mineral and vitamin deficiencies can accelerate the mitochondrial decay of aging. Molecular Aspects in Medicine 2005; 26(4-5):363-78.

35. Bottoni A, Rodrigues RC, Celano RMG. Papel da nutrição na cicatrização. Revista Ciências em Saúde 2011; 1(1):98-103.

36. Sato Y. Abnormal bone and calcium metabolism in patients after stroke. Archives of Physical Medicine and Rehabilitation 2000; 81(1):117-121.

37. Okada A, Ohshima H, Itoh Y, Yasui T, Tozawa K, Kohri K. Risk of renal stone formation induced by long-term bed rest could be decreased by premedication with bisphosphonate and increased by resistive exercise. International. Journal of Urology 2008; 15(7):630-5.

38. He K, Song Y, Belin RJ, Chen Y. Magnesium intake and the metabolic syndrome: epidemiologic evidence to date. Journal of the CardioMetabolic Syndrome 2006; 1(5):351-355.

39. Rayman MP. Selenium and human health. The Lancet 2012; 379(9822):1256-1268.

40. Mente A, O’Donnell MJ, Yusuf S. The population risk of dietary salt excess are greatly exaggerated. Canadian Journal of Cardiology 2014; 30(5):507-512.

41. He JF, Li J, MacGregor GA. Effect of longer term modest salt reduction on blood pressure: Cochrane systematic review and meta-analysis of randomised trials. BMJ 2013; 346:f1325.

42. Drewnowski A, Maillot M, Rehm C. Reducing the sodium-potassium ratio in the US diet: a challenge for public health. American Journal of Clinical Nutrition 2012; 96:439-444.

43. Yang Q, Liu T, Kuklina EV, Flanders WD, Hong Y, Gillespie C, et al. Sodium and potassium intake and mortality among US adults: prospective data from the Third National Health and Nutrition Examination Survey. Archives of Internal Medicine 2011; 171(13):1183-1191.

Recebido: $14 / 4 / 2014$

Revisado: 29/5/2014

Aprovado: 06/7/2014 
